\title{
Various Aspects of the Contrast Modalities of Modulated Beam Imaging
}

\author{
Markus Strobl $\left.\right|^{1,2, a}$ * \\ ${ }^{1}$ Paul Scherrer Institut (PSI), Laboratory for Neutron Scattering and Imaging, 5232 Villigen, \\ Switzerland \\ ${ }^{2}$ University of Copenhagen, Niels Bohr Institute, Denmark \\ amarkus.strobl@psi.ch \\ * corresponding author
}

Keywords: Imaging, Grating Interferometry, Attenuation, Differential Phase Contrast, Dark-Field Contrast

\begin{abstract}
Since the introduction of grating interferometers to imaging, in addition to attenuation contrast, differential phase and later also dark-field contrast imaging have been explored intensely. However, in particular with dark-field contrast imaging, imaging entered into a new domain, i.e. the scattering from sub-image-resolution structures. This has led to the need to expand the horizon of considered interactions into the reciprocal space domain of small angle scattering, not necessarily familiar in real space imaging. Correspondingly, description and interpretation and finally quantitative analyses lacked somewhat behind of on the other hand qualitatively invaluable results. Against this background all modalities measured in modulated beam imaging experiments, namely attenuation, differential phase and dark-field contrast, shall be given some additional attention. It will be undertaken to draw a clear picture of analogies, of contrast formation and consequences to interpretation and information content.
\end{abstract}

\section{Introduction}

In imaging with a spatially modulated beam, the beam modulation is superimposed to the image of the sample. Assuming a one-dimensional modulation, it can in general be described by a simple sinusoidal intensity modulation function with a period $\mathrm{p}$ superimposed to the conventional radiographic projection $\mathrm{a}(\mathrm{x})$ as

$$
\mathrm{I}(\mathrm{x})=\mathrm{a}(\mathrm{x})+\mathrm{b}(\mathrm{x}) \sin (2 \pi \mathrm{x} / \mathrm{p}+\Delta \phi(\mathrm{x}))
$$

Generally, three images of different contrast modalities are/can be deduced from such projection data. Attenuation contrast, manifested as the local average count over one period, or in other words the offset of the modulation a(x); differential phase contrast, the local relative phase of the modulation compared to the undisturbed phase of an empty beam measurement $\Delta \phi(\mathrm{x})$; finally, dark field contrast, the relative local amplitude of the modulation $b(x) / a(x)$. For simplicity it is considered here, that the modulation period is smaller than the spatial resolution of the detector, in which case it is conventionally measured by a scanning procedure and an analyser grating matching the modulation period. This provides a separate modulation in each pixel of an image recorded. Therefore, we can consider the principal spatial resolution limit for all three signals at best twice the pixel size. Note that therefore henceforth $\mathrm{x}$ shall be the transversal parameter on the length scale of the pixel resolution. Instead of describing the subpixel modulation every pixel is assumed to have well defined parameters $\mathrm{a}(\mathrm{x}), \mathrm{b}(\mathrm{x})$ and $\Delta \phi(\mathrm{x})$. 
Sufficient coherence appears to be a prerequisite to exploit beam modulation through grating interferometry based on the Talbot Lau effect [1], because the interference pattern of waves induced by a grating establishes the spatial beam modulation. Hence, the period of that grating implies the coherence requirement. For geometries with insufficient coherence of the beam at the grating the requirement is efficiently circumvented or solved by creating a partially coherent beam with a source grating, i.e. several beams with a coherence satisfying the requirement and superimposing through geometrical alignment constructively at the detector, respectively the analyzer grating. Coherence is here interpreted as the spatial coherence width of a beam at the grating generating the modulation achieved through collimation by a slit (slit grating, i.e. source grating) at a significant distance upstream. Different realizations and details of the set-ups shall not be discussed here but can be found in literature. It has to be noted that meanwhile a great number of variations exists, but the principles generally stay the same for modulated beam techniques. Here we shall focus on the principles of the detected signals and their origins.

\section{Attenuation contrast}

Attenuation contrast is the most common and conventional as well as apparently best known contrast mechanism. It can in most cases be simply described by the Beer-Lambert law [2]

$$
a(x)=a_{0}(x) e^{-\int \mu(x, y, \lambda) d y}
$$

Including the geometry of the beam and the sample as well as the energy, hence, also wavelength $\lambda$, dependent linear attenuation coefficient $\mu$. Note, that the geometrical description is limited to the plane of the beam direction $\mathrm{y}$ and the transversal coordinate $\mathrm{x}$, as for a beam assumed parallel this is sufficient, even for tomographic considerations. In addition, this will also suffice for the principle considerations on other grating based contrast signals, due to the grating symmetry. It is useful to present the well-known Beer Lambert equation as it will be a point of reference and analogy for the other, one might refer to as novel, contrast mechanisms. For this reason we also look a bit closer at the attenuation coefficient and what it is constituted of. For neutrons the linear attenuation coefficient $\mu$, a reciprocal length, can be expressed as the product of the neutron cross section $\sigma(\lambda)$ and the particle density $\mathrm{N}$

$$
\mu=\sigma \mathrm{N}
$$

In general the linear attenuation coefficient is assumed orientation invariant, a density property. However, attenuation is constituted not only by absorption, as very often wrongly assumed or inconsiderately expressed, but also by scattering. Correspondingly $\sigma=\sigma_{\mathrm{s}}+\sigma_{\mathrm{a}}$ features a scattering and absorption term, respectively. Scattering which does not exempt directions close to the forward direction can lead to significant bias in measured cross sections, depending on the measurement geometry, in particular the sample to detector distance [3-12]. More interestingly, for neutron wavelengths utilized in grating interferometric imaging the scattering term, or even the total cross section, for crystalline materials is in many cases dominated by coherent elastic scattering from the crystal lattice. This implies that depending on crystallite sizes and microstructural isotropy, the linear attenuation coefficient can become orientation dependent as the coherent elastic part of $\sigma_{s}$ can then be written as

$$
\sigma_{\text {coh,elas }}(\lambda, \Omega)=\sum_{n}\left[\frac{(2 \pi)^{3} N}{2 k \tau_{h k l}} \sum_{h k l}\left|F_{h k l}\right|^{2} \delta_{D}\left(\tau_{h k l}^{2}-2 k \tau_{h k l} \sin \theta_{n, h k l}\right)\right]
$$


where $\mathrm{k}=2 \pi / \lambda$ is the modulus of the wavevector and correspondingly $\tau=2 \pi / \mathrm{d}$ is the length of the reciprocal lattice vector. $\Omega$ symbolizes the sample orientation in space, $\mathrm{n}$ is the number of individual crystal grains, while $\mathrm{F}_{\mathrm{hkl}}$ is the structure factor and $\delta_{\mathrm{D}}$ is representing the Dirac Delta function in expressing the Bragg condition with $\theta$ being the Bragg angle. A fact that leads to anisotropic behavior of samples as attenuation of certain volumes will differ for different projection angles e.g. due to texture effects (Fig. 1). In particular for grain mapping with neutrons from the transmission data [13] but also other investigations of local crystalline structure [14], these orientation dependent attenuation effects can be exploited while they are ignored in most standard applications. For 3D phase mapping for example, but given the example in Fig. 1 below, even in conventional white beam tomography, such orientation dependent effects can lead to significant bias, which needs to be considered.

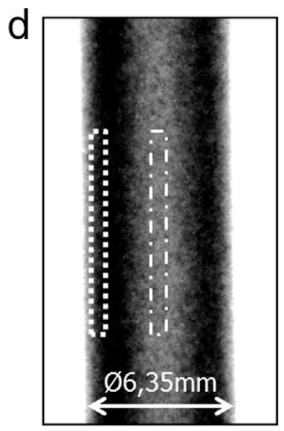

$0^{\circ}$

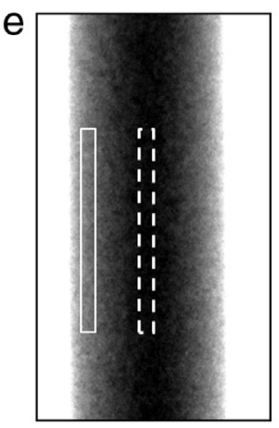

$30^{\circ}$

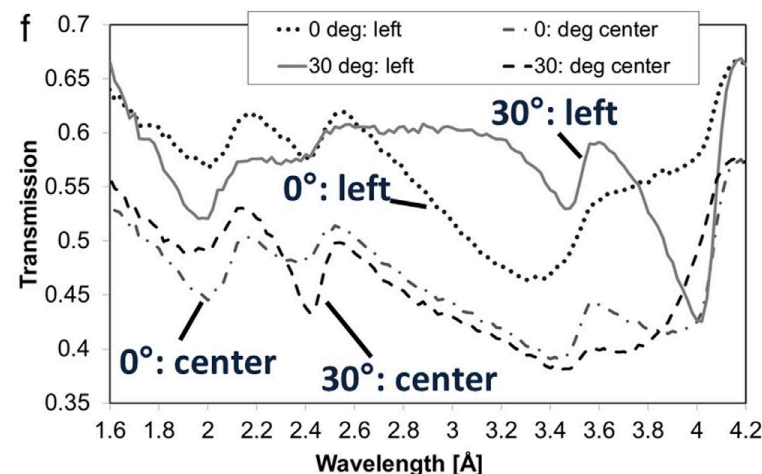

Wavelength $[\AA]$

Fig. 1 Significantly textured but otherwise homogeneous cylindrical steel sample exposed at two different projection angles (left) and wavelength dependent transmission profiles according to the highlighted areas in the field of view on the left hand side radiographies (right).

\section{Differential phase contrast}

The second contrast modality is referred to as differential phase contrast, sometimes also just phase contrast. In contrast to the transmitted intensity, the phase of the radiation is not accessible straightforwardly. It constitutes a fundamentally different feature of the applied radiation than the detected intensity. The measurement of phase relations is the domain of interferometry and in particular neutron Mach-Zehnder interferometers [15] have been used for such purpose. In some cases such interferometers were also utilized for imaging based on phase shifts induced by samples with both x-rays [16] and neutrons [17]. However, due to the significant coherence and stability requirements of perfect crystal interferometers phase contrast became relevant for broad application, especially with $\mathrm{x}$-rays, only later with the introduction of differential phase contrast $[18,19]$ and propagation based techniques $[20,21]$. These techniques exploit the fact, that phase objects distort the wavefront and the respective locally induced angular deviations are measured directly with significant angular resolution or through the induced spatial intensity shift at different detector distances, respectively. The angular deviation induced, as described by refraction, corresponds to the induced local phase gradient $\nabla \varphi$, lending the name differential phase to this contrast modality. The rather small angles (arcsec) were first resolved by the use of analyser crystals in monochromator/analyser arrangements (i.e. perfect crystal double crystal arrangements), and methods called Schlieren imaging, diffraction enhanced or refraction contrast imaging [18,19,22-26]. However, grating interferometers introduced around the millennium [2731] outperformed the other methods significantly due to relaxed coherence requirements, both in real and momentum space. The Talbot Lau grating interferometers resolve small angles due to 
induced spatial beam modulation, for which meanwhile several methods are available. Interestingly in grating interferometers the differential phase translates into a phase shift of the real space modulation according to

$$
\nabla j=\partial j / \partial x \propto \operatorname{Df}(x)
$$

The thus measured orientation dependent refraction, i.e. differential phase (Fig. 2 mid), can easily be transferred into the orientation independent phase contrast (Fig. 2 right) related to the real part of the refractive index $\delta$ by integration

$$
\varphi(x)=\int \delta(x, y) d y \propto \int_{0}^{x} \Delta \phi(x) d x
$$

Note that orientation dependence and independence here refers to the local interaction. The relation in eq. 6 provides a straightforward opportunity for tomographic reconstruction of the refractive index distribution $\delta(\mathrm{x}, \mathrm{y})$, a scalar quantity. This constitutes an analogy to the linear attenuation coefficient $\mu$ or the imaginary part of the refractive index $\beta$ retrieved in conventional attenuation or absorption contrast imaging, respectively (Fig. 2). The local orientation dependence of the measured refraction effects is neutralized through the integral which turns the differential phase into a phase contrast signal.

Because descriptions appear ambiguous and misleading sometimes it shall be clarified that refraction, i.e. the differential phase effect does not require specific coherence, due to the differential nature, and can be described by geometrical optics. Independent of the fact that differential phase effects do not necessarily preserve coherence characteristics of the affected beam, refraction in general also causes loss of modulation visibility and hence a dark-field contrast signal. In general differential phase and dark-field contrast are very closely related and interlinked in parts, but not equivalent. Clear distinction and understanding of the measured nature of interaction is key for correct interpretations of results in this regime as shall be discussed in the following section on dark-field contrast.
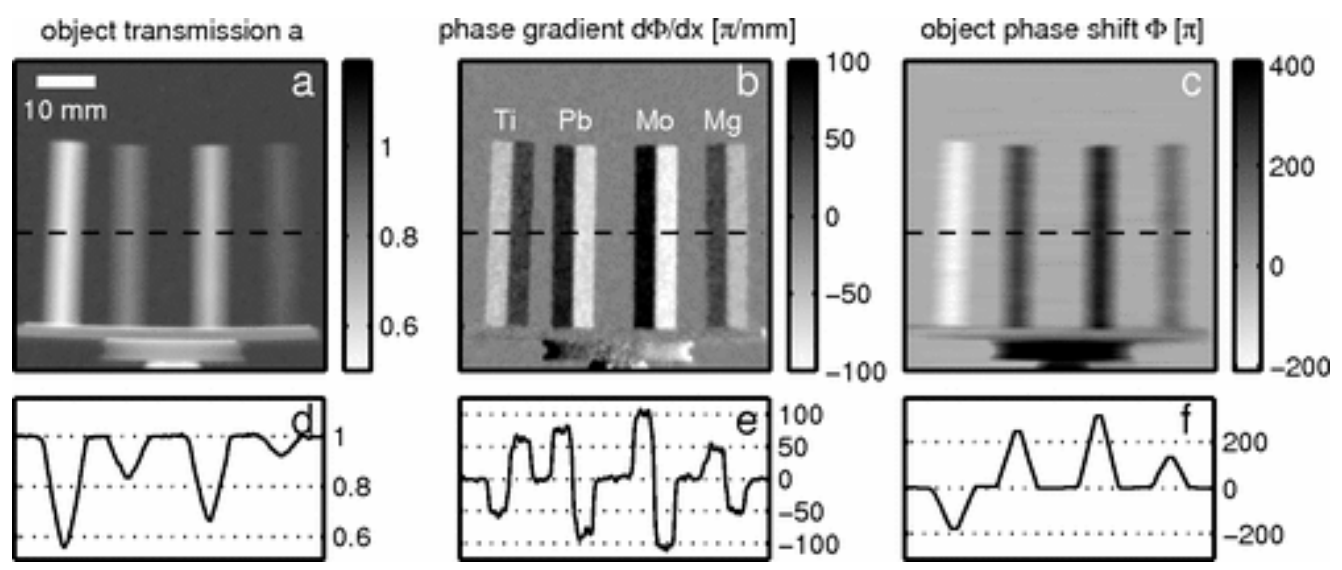

Fig. 2 This reprint with permission of AIP from Ref. [31] illustrates the analogy of attenuation and phase contrast (left and right), as well as the relation of differential phase contrast (mid) and phase contrast through transversal integration (right).

Dark-field contrast

The third contrast modality measured in a grating interferometer set-up for imaging has maybe caused the highest amount of ambiguity. It is based on the measure of the local modulation amplitude $\mathrm{b}(\mathrm{x})$, which needs to be normalized by the attenuation $\mathrm{a}(\mathrm{x})$ or simply expressed as the 
visibility $\mathrm{V}=\left(\mathrm{I}_{\max }-\mathrm{I}_{\min }\right) /\left(\mathrm{I}_{\max }+\mathrm{I}_{\min }\right)=\mathrm{b}(\mathrm{x}) / \mathrm{a}(\mathrm{x})$. Naturally, only the amplitude relative to the transmission constitutes a useful independent measure. Again, historically this kind of contrast has been utilized already long before grating interferometers were introduced in imaging with xrays and neutrons, but was referred to with different terminology. In fact first neutron examples can be found already in the 1980ties [17,32] for magnetic structures, which for neutrons still constitute one of the main applications. In x-ray imaging one could consider the first works to be described in Ref [33,34] while first tomography was reported with x-rays in [35] shortly after first neutron dark-field tomography, back then referred to as USANS tomography, was reported [36].

While in summary the dark-field contrast measured always relates to an additional local angular spread of the incident beam, the contrast's origin has so far been attributed to (ultra) small angle scattering ((U)SAS)[34,36,37,38], Fresnel diffraction [17], refraction [37,39], incoherent scattering [40] and decoherence [41,42]. The term decoherence, which is generally in physics defined as the "process in which a system's behaviour changes from that which can be explained by quantum mechanics to that which can be explained by classical mechanics"[43], might intend to mean a certain loss of coherence, though e.g. small angle scattering and Fresnel diffraction are coherent scattering processes and refraction does e.g. in the differential phase contrast not necessarily alter the coherence significantly either.

However, a loss of modulation visibility can have a number of reasons, not necessarily related to a loss of coherence. It is e.g. obvious that a slight alteration of a three grating set-up where multiple beams and hence interference patterns are superimposed constructively, could lead to a destructive superposition. In contrast to a loss of interference of wavefunctions (e.g. through a loss of coherence) this is, however, a classical superposition adding intensities, not phase dependent amplitudes of a wavefunction.
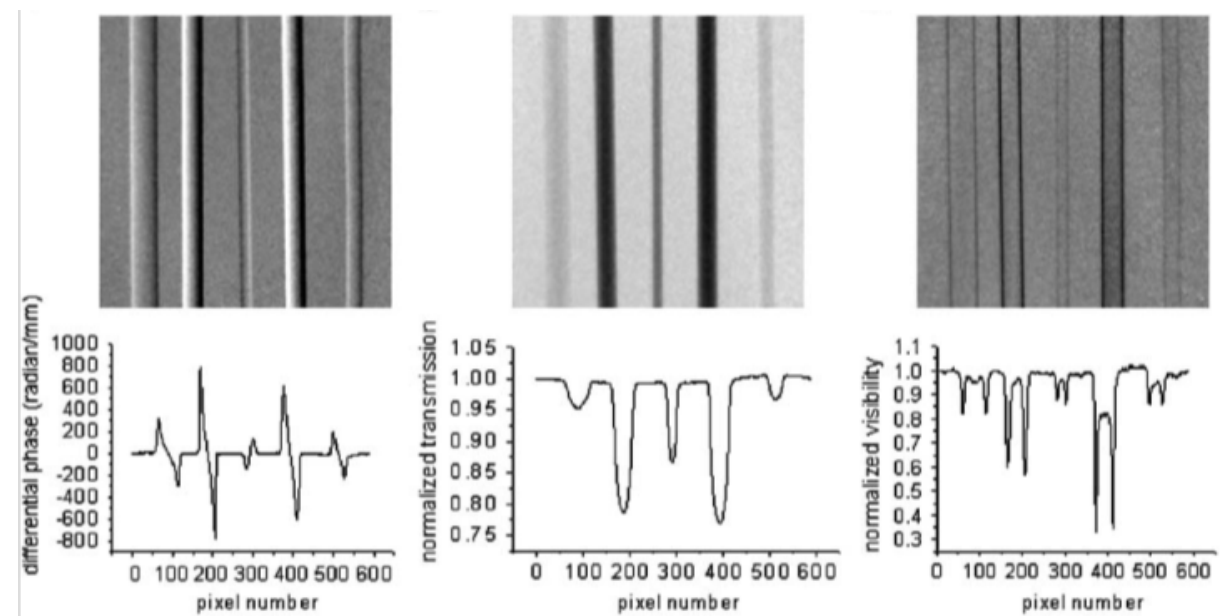

Fig. 3 Differential phase, attenuation and dark-field contrast profiles of a number of cylindric samples (reprinted with permission from [62])

Bearing such classical addition of interference patterns in mind, it is useful to reconsider the conditions of differential phase contrast, where within the spatial detector resolution a phase shift of modulation pattern is expected in one defined direction and magnitude. However, within a pixel there are or might be a number of modulation periods and a structure below this resolution limit might refract the beam to different directions, causing one part of the modulations within a pixel to shift significantly different to the other(s). The integrated measurement of the 
modulation within a pixel, however, will cause a loss of modulation amplitude, which in the actual pattern might not even exist. (It will indeed always exist in a certain region, where affected and non-affected or differently affected parts of the modulation overlap as well as due to divergence and intrinsic spatial resolution limitations). However, the actual coherence characteristics cannot depend on the pixel size, and hitherto a simple loss of coherence or even decoherence argument can obviously not apply to explain the measured result in general.

With this refraction argument it already becomes clear that, similar to attenuation contrast which can consist of scattering and/or absorption, the measured loss of amplitude referred to as darkfield contrast, does not yet allow a unique interpretation of the physical process measured. Correspondingly, it has been expressed already in the initial neutron grating dark-field publication [37] that "In the region of sharp edges refracted and non-refracted contributions are superimposed and hence decrease the fringe visibility. This accounts for enhanced contrast in the reconstruction at corners and curves, i.e., wherever an edge is not well defined due to limited resolution", and in Ref [44] it is stated, that "measurements show that due to the limited spatial resolution, refraction, i.e., differential phase contrast, also always affects the dark-field image"(compare Fig. 3). And therefore :"Different origins of the dark-field signal have to be taken into account, namely, ultra-small-angle scattering and refraction, which might complicate a straightforward interpretation of specific results with respect to the quantification of the size of inhomogeneities and defects.” In X-ray dark-field imaging consciousness is raised only recently by a dedicated work [38] which similarly reads "Results of numerical calculations for monochromatic X-rays show that an unresolvable sharp edge generates not only differentialphase contrast but also visibility contrast."

To some extent, hence, the dark-field contrast measured due to resolution limits can be compared to attenuation values biased by resolution, either at interfaces or when structures are significantly below resolution and hence affect the measured density, where one cannot distinguish whether the overall density changes in this area or whether the very same material with the same density includes distinct structural features like e.g. pores, that are not resolved. (In fact a differentiation of the attenuation contrast could provide a contrast similar to differential phase contrast at edges, including the orientation dependence - a differential attenuation contrast so to speak (compare Fig. 2). However, when assuming refraction (i.e. differential phase contrast) and small angle scattering, referred to in most cases, to be the main sources of darkfield contrast, it is required to take a deeper look into these and how they are distinguished.

In general, small angle scattering is described by scattering theory [45] and in accordance to the used kinematic or first Born approximation [46] phase effects are neglected. This means in small angle scattering it is assumed that phase shifts between scattering centers are negligible, due to the small size and weak phase interaction. In ultra-small angle scattering, where structures can have sizes in the micrometer range, this assumption does not necessarily hold anymore, and interestingly refraction effects are measured in the very same angular range [47]. While in small angle scattering, described by a coherent superposition of waves scattered at different locations within an object, the subsequent angular distribution of the emerging interference, i.e. scattering pattern, is strongly size and shape dependent, the angular pattern of refraction is only shape dependent. Generally it is assumed, that when the approximation of negligible phase shifts does not hold anymore the SAS description breaks down and refraction dominates. Correspondingly it is to be expected, that a different quantification in terms of phase objects is required in this case. As this is not a problem only to dark-field imaging but also scattering experiments in this regime a number of works and investigations exist. Earlier works include N. F. Berk and K. A. Hardman-Rhyn [48], who come to the conclusion "that strong multiple scattering, as in very thick samples, tends to render beam broadening insensitive to the cross over from diffractive to 
refractive single-particle scattering." But "On the other hand, (...), say for scattering from large particles dilutely dispersed in thin samples, departures from the diffractive limit should be important." More recent considerations in particular for neutron refraction measured in USAS can be found e.g. in Ref [49,50].

Based on this discussion some caution is required characterizing dark-field contrast based on a "material constant" $\varepsilon$ referred to as "linear diffusion coefficient" [51,52]. However, it is clear that the visibility due to convolution of the resulting angular profile with the modulation function [37] can be described by an exponential contrast behavior based on a path integral accounting for subsequent (multiple) interactions approximated by Gaussian angular distributions [36,53]. In analogy to attenuation and Beer Lamberts law this can hence be written as

$$
V(x)=V_{0}(x) e^{\int \varepsilon(x, y, \lambda) d y}
$$

and consequently enables tomographic reconstruction analogue to attenuation contrast tomography [35,36,37,51].

For small angle scattering the coefficient $\varepsilon$ could be specified as [54]

$$
\varepsilon(r)=\Sigma_{\text {SAS }}(G(r)-1)
$$

where $\Sigma_{\text {SAS }}$ is the total small angle scattering cross section, dependent on characteristic structure sizes, volume fractions and scattering contrast (for neutrons scattering length density contrast, for X-rays electron density contrast) and $\mathrm{G}(\mathrm{r})$ is the projected real space correlation function of the scattering structure [54-56]. The microscopic real space parameter $\mathrm{r}$ probed in a measurement is equivalent to the autocorrelation length parameter

$$
\xi=\lambda \mathrm{L}_{S} / \mathrm{p}
$$

of the applied modulated beam measurement [54], where $L_{S}$ is the sample to modulation detection distance.

It is worth noting, that the measured effects are described by simple superpositions of intensities and hence by geometric optics. While the interference pattern created by the respective grating and the small angle scattering have to be based on coherent wave optics, the grating and the scattering sample are not in any coherent correlation, and hence the results from both interference effects can be considered separately and combined through geometrical optics i.e. through intensity superposition [37,54]. Hence, coherence and decoherence considerations appear apart from the separated local coherent processes obsolete in this context.

However, there is a contribution to dark-field contrast from incoherent scattering indeed. Like in SAS incoherent scattering has to be assumed to generate a background signal, for neutrons in particular when hydrogen is involved, e.g. for particles in aqueous solution. The fraction of incoherently scattered neutrons will not contribute to the interference modulation on the detector, but such way forward scattered neutrons will contribute to the transmission $\mathrm{a}(\mathrm{x})$, while the rest will contribute to the attenuation $(1-a(x)) / a_{0}(x)$. Corresponding corrections required for the quantification of the coherent SAS signal have been taken into account for various quantitative neutron works [40,57].

For refraction contributions to the dark-field contrast no general model is available and so far it is treated phenomenologically and qualitatively, i.e. as indicator for irregularities beyond spatial resolution that are not further analyzed. However, in analogous (U)SAS methods without 
spatial resolution some considerations for regular structures exist $[49,50]$, which can directly be transferred. For example for aligned cylindrical shapes with axis parallel to the modulation

$$
\mathrm{V}(\mathrm{r}) / \mathrm{V}_{0}(\mathrm{r})=2 \delta \mathrm{r} \mathrm{K}_{1}(\lambda \mathrm{r}) / \pi \lambda
$$

has been found [50], where $K_{1}$ is the first order modified Bessel function of second kind. Nevertheless, in most cases so far, where only the identification, but not the quantification and characterization, of defects beyond direct spatial resolution is in the center of attention, this will not play a major role. Whether equ.(7) applies in such cases, and how reliable tomographic reconstructions from such kind of artifacts is, requires to be clarified yet as well. However, a significant difference between refraction and SAS has to be considered carefully in all assumptions of detected effects and contributions. In particular it has to be regarded, that while SAS only concerns a small fraction of incident radiation and hence requires a certain volume (fraction) to be measureable in particular in transmission, phase shift and hence refraction at inclined interfaces affect all passing radiation. Hence, differential phase effects enable detection of single individual structures (of sizes competitive with, i.e. of significant fraction of the resolution limit), while SAS requires a large number and density of similar structures, hence significantly beyond the resolution limit to provide an effective signal. A straightforward distinction from the measurement does not appear obvious and hence attempts for quantitative structure determination through dark field imaging still require a priori knowledge and/or additional means of characterization. An orientation dependence is possible for both signals, depending on the geometry of the structures, but is not necessarily given, like e.g. in the cases of dark-field signal from refraction at macroscopic edges. A careful consideration of expected structure sizes and corresponding phase shifts induced appears absolutely necessary.

For neutrons in particular the refractive nature of dark-field contrast has another dimension and plays a central role for the maybe most exploited dark-field imaging application, namely of magnetic structures $[17,41,58-60]$. The magnetic field contributes to the real part of the refractive index for neutrons due to their magnetic moment $\mu_{\mathrm{n}}$ coupled to the neutron spin. The neutron spin aligns parallel and antiparallel to an external magnetic field and the neutron correspondingly gains or looses momentum in the field. At interfaces with a strong magnetic field gradient, where the spin cannot adiabatically follow the magnetic field thus refraction takes place according to the refractive index contribution

$$
\delta_{\mathrm{B}}= \pm 2 \mu_{\mathrm{n}} \mathrm{mB} \lambda^{2} / \mathrm{h}^{2}
$$

where $\mathrm{m}$ is the mass of the neutron and $\mathrm{h}$ the Planck constant. Due to the fact that for an unpolarised beam this implies symmetric refraction from the same local structure to opposite directions for the two spin eigenstates, this will in such case always lead to dark-field contrast through the superposition of opposite differential phase contrast signals from the same location. This has already been measured in interferometers and analyser based set-ups $[17,58]$ visualizing domain walls. With a polarized beam however, the undisturbed local differential phase contrast can be measured and has been demonstrated to be usable for the reconstruction of strong magnetic fields through the magnetic refractive index in full analogy to differential phase contrast tomography [61]. 


\section{References}

[1] Henry Fox Talbot: LXXVI. Facts relating to optical science. No IV. In: The London Edinburgh Philosophical Magazine and Journal of Science. Band 9, Nr 56, 1836, Par.2 Experiments on Diffraction, pp 401. https://doi.org/10.1080/14786443608649032

[2] Pierre Bouguer: Essai d’optique, Sur la gradation de la lumiere. Claude Jombert, Paris 1792, pp 164

[3] P. M. Joseph, “'The effects of scatter in x-ray computed tomography,” Med. Phys. 9 (1982) 464-472. https://doi.org/10.1118/1.595111

[4] G. H. Glover, “'Compton scatter effects in CT reconstructions,’’ Med. Phys. 9 (1982) 860867. https://doi.org/10.1118/1.595197

[5] L. A. Love and R. A. Kruger, "'Scatter estimation for a digital radio- graphic system using convolution filtering,’’ Med. Phys. 14 (1987) 178 - 185. https://doi.org/10.1118/1.596126

[6] J. A. Seibert and J. M. Boone, “X-ray scatter removal by deconvolution,’’ Med. Phys. 15 (1988) 567-575. https://doi.org/10.1118/1.596208

[7] D. G. Kruger, F. Zink, W. W. Peppler, D. L. Ergun, and C. A. Mistretta, “'A regional convolution kernel algorithm for scatter correction in dual- energy images: Comparison to single-kernel algorithms,’’ Med. Phys. 21 (1994) 175-184. https://doi.org/10.1118/1.597297

[8] Kardjilov, N., de Beer, F., Hassanein, R., Lehmann, E. \& Vontobel, P. Nucl. Instrum. Methods Phys. Res. A, 542 (2005) 336-341. https://doi.org/10.1016/j.nima.2005.01.159

[9] Pekula, N., Heller, K., Chuang, P. A., Turhan, A., Mench, M. M., Brenizer, J. S. \&Uenlue , K., Nucl. Instrum. Methods Phys. Res. A, 542 (2005) 134-141.

https://doi.org/10.1016/j.nima.2005.01.090

[10] Hassanein, R., Lehmann, E. \& Vontobel, P., Nucl. Instrum. Methods Phys. Res. A, 542 (2005) 353-360. https://doi.org/10.1016/j.nima.2005.01.161

[11] Tremsin, A. S., Kardjilov, N., Dawson, M., Strobl, M., Manke, I., McPhate, J. B., Vallerga, J. V., Siegmund, O. H. W. \& Feller, W. B., Nucl. Instrum. Methods Phys. Res. A, 651 (2011) 145-148. https://doi.org/10.1016/j.nima.2011.01.066

[12] M. Raventos, E. H. Lehmann, M. Boin, M. Morgano, J. Hovind, R. Harti, J. Valsecchi, A. Kaestner, C. Carminati, P. Boillat, P. Trtik, F. Schmid, M. Siegwart, D. Mannes, M. Strobl and C. Gruenzweig, A Monte Carlo approach for scattering correction towards quantitative neutron imaging of polycrystals, J. Appl. Cryst. 51 (2018). https://doi.org/10.1107/S1600576718001607

[13] A. Cereser, M. Strobl, S. A. Hall, A. Steuwer, R. Kiyanagi, A. S. Tremsin, E. B. Knudsen, T. Shinohara, P. K. Willendrup, A. Bastos da Silva Fanta, S. Iyengar, P. M. Larsen, T.

Hanashima, T. Moyoshi, P. M. Kadletz, P. Krooß, T. Niendorf, M. Sales, W. W. Schmahl, and S. Schmidt Time-of-Flight Three Dimensional Neutron Diffraction in Transmission Mode for Mapping Crystal Grain Structures, Scientific Reports 7 (2017) 9561.

https://doi.org/10.1038/s41598-017-09717-w

[14] Robin Woracek, Javier Santisteban, Anna Fedrigo, Markus Strobl, Diffraction in neutron imaging-A review, Nucl. Inst. Meth. A 878 (2018) 141-158.

https://doi.org/10.1016/j.nima.2017.07.040 
[15] Ludwig Zehnder: Ein neuer Interferenzrefraktor. In: Zeitschrift fuer Instrumentenkunde. Nr. 11, 1891, pp 275; Ludwig Mach: Ueber einen Interferenzrefraktor. In: Zeitschrift fuer Instrumentenkunde. Nr. 12, 1892, pp 89

[16] M. Ando and S. Hosoya, in Proc. $6^{\text {th }}$ Intern. Conf. on X-ray Optics and Microanalyses, eds. G. Shinoda, K. Kohra and T. Ichinokawa (Univ. of Tokyo Press, Tokyo, 1072) 63

[17] M. Schlenker, W. Bauspiess, W. Graeff, U. Bonse, H. Rauch, J. Magn. Magn. Mat.15-18 (1980) 1507. https://doi.org/10.1016/0304-8853(80)90387-X

[18] K. Goetz, M.P. Kalashnikov, Yu. A. Mikhailov, G. V., Sklizkov, S. I. Fedotov, E. Foerster,P., Zaumseil: Preprint Nr. 159, FIAN UdSSR (1978)

[19] K.M. Podurets et al. Zh. Tekh. Fiz.59 (1989) 115-121

[20] Snigirev, A., Snigireva, I., Kohn, V., Kuznetsov, S. \& Schelokov, I. On the possibilities of $\mathrm{x}$-ray phase contrast microimaging by coherent high-energy synchrotron radiation. Rev. Sci. Instrum. 66 (1995) 5486-5492 . https://doi.org/10.1063/1.1146073

[21] B. E. Allman, P. J. McMahon, K. A. Nugent, D. Paganin, D. L. Jacobson, M. Arif \& S. A. Werner, Imaging: Phase radiography with neutrons, Nature 408 (2000) 158-159.

https://doi.org/10.1038/35041626

[22] Ingal, V. N. \& Beliaevskaya, E. A. X-ray plane-wave topography observation of the phase contrast from a non-crystalline object. J. Phys. D 28 (1995) 2314-2317. https://doi.org/10.1088/0022-3727/28/11/012

[23] Davis, T. J., Gao, D., Gureyev, T. E., Stevenson, A. W. \& Wilkins, S. W. Phase-contrast imaging of weakly absorbing materials using hard X-rays. Nature 373 (1995) 595-598. https://doi.org/10.1038/373595a0

[24] Chapman, L. D. et al. Diffraction enhanced x-ray imaging. Phys. Med. Biol. 42 (1997) 2015-2025. https://doi.org/10.1088/0031-9155/42/11/001

[25] W. Treimer, M. Strobl, A. Hilger, C. Seifert, U. Feye-Treimer, Refraction as imaging signal for computerized (neutron) tomography, Applied Physics Letters, 83, 2 (2003) 398-400. https://doi.org/10.1063/1.1591066

[26] M. Strobl, W. Treimer, A. Hilger, First realisation of a three-dimensional refraction contrast computerised neutron tomography, Nucl. Instr. Meth. B 222, 3-4 (2004) 653-658.

https://doi.org/10.1016/j.nimb.2004.02.029

[27] J. F. Clauser, U.S. Patent No. 5,812,629 (1998);

[28] A. Momose, S. Kawamoto, I. Koyama, Y. Hamaishi, K. Takai and Y. Suzuki: Jpn. J. Appl. Phys. 42 (2003) L866. https://doi.org/10.1143/JJAP.42.L866

[29] T. Weitkamp, B. Noehammer, A. Diaz and C. David: Appl. Phys. Lett. 86 (2005) 054101. https://doi.org/10.1063/1.1857066

[30] F. Pfeiffer, T. Weitkamp, O. Bunk, and C. David, Nature Phys. 2, 258 (2006).

https://doi.org/10.1038/nphys265

[31] F. Pfeiffer, C. Gruenzweig, O. Bunk, G. Frei, E. Lehmann, and C. David, Phys. Rev. Lett. 96, 215505 (2006). https://doi.org/10.1103/PhysRevLett.96.215505 
[32] K.M. Podurets et al Physica B 156 \& 157 (1989) 694-697. https://doi.org/10.1016/09214526(89)90766-7

[33] Pagot, E. et al. A method to extract quantitative information in analyzer-based X-ray phase contrast imaging. Appl. Phys. Lett. 82, 3421-3423 (2003). https://doi.org/10.1063/1.1575508

[34] L. Rigon, H. -J. Besch, F. Arfelli, R.-H. Menk, G. Heitner, and H. P.Besch, “A new DEI algorithm capable of investigating sub-pixel structures,”J. Phys. D 36, 107-112 (2003). https://doi.org/10.1088/0022-3727/36/10A/322

[35] J.G. Brankov, M.N. Wernick, Y. Yang, J. Li , C. Muehleman, Z. Zhong and M.A. Anastasio A computed tomography implementation of multiple-image radiography Med. Phys. 33 (2006) 278. https://doi.org/10.1118/1.2150788

[36] M. Strobl, W. Treimer, A. Hilger, Small angle scattering signals for (neutron) computerized tomography Appl. Phys. Lett., 85, 3 (2004) 488-490. https://doi.org/10.1063/1.1774253

[37] M. Strobl, C. Grünzweig, A. Hilger, I. Manke, N. Kardjilov, C. David, F. Pfeiffer, Neutron dark-field tomography, Phys. Rev. Lett. 101, 123902 (2008).

https://doi.org/10.1103/PhysRevLett.101.123902

[38] F. Pfeiffer, M. Bech, O. Bunk, P. Kraft, E. F. Eikenberry, C. Broennimann, C. Gruenzweig and C. David, Hard-X-ray dark-field imaging using a grating interferometer, Nat. Mat. 7 (2008) 134. https://doi.org/10.1038/nmat2096

[39] W. Yashiro et al., Opt. Exp. 9233, 23 (2015) 7. https://doi.org/10.1364/OE.23.009233

[40] B. Betz, R. P. Harti, M. Strobl, J. Hovind, A. Kaestner, E. Lehmann, H. Van Swygenhoven and C. Grünzweig, Quantification of the sensitivity range in neutron dark-field imaging, Rev. Sci. Instrum. 86, 123704 (2015). https://doi.org/10.1063/1.4937616

[41] 5. C. Grünzweig, C. David, O. Bunk, M. Dierolf, G. Frei, G. Kühne, J. Kohlbrecher, R. Schäfer, P. Lejcek, H. Rønnow, and F. Pfeiffer, Neutron decoherence imaging for visualizing bulk magnetic domain structures, Phys. Rev. Lett. 101, 025504 (2008).

https://doi.org/10.1103/PhysRevLett.101.025504

[42] T. Lauridsen, M. Willner, M. Bech, F. Pfeiffer, R. Feidenhans'l Detection of sub-pixel fractures in X-ray dark-field tomography Applied Physics A 121 (2015).

https://doi.org/10.1007/s00339-015-9496-2

[43] http://www.dictionary.com/browse/decoherence

[44] A. Hilger, N. Kardjilov, T. Kandemir, I. Manke, and J. Banhart, D. Penumadu, A. Manescu, M. Strobl, Revealing micro-structural inhomogeneities with dark-field neutron imaging, J. Appl. Phys. 107, 036101 (2010). https://doi.org/10.1063/1.3298440

[45] Feigin, L. \& Svergun, D. Structure Analysis by Small-Angle X-ray and Neutron Scattering (New York Plenum Press, 1987).

[46] Born, Max Quantenmechanik der Stossvorgänge, Zeitschrift für Physik. 38: 803 (1926). https://doi.org/10.1007/BF01397184

[47] W. Treimer, M. Strobl, A. Hilger Observation of edge refraction in ultra small angle neutron scattering Phys. Lett. A 305, 1-2 (2002) 87-92. https://doi.org/10.1016/S0375-9601(02)01391-9 
[48] N.F. Berk and K. A. Hardman-Rhyn, Analysis of SAS Data Dominated by Multiple Scattering, J. Appl. Cryst. 21 (1988) 645-651. https://doi.org/10.1107/S0021889888004054

[49] Victor-O. de Haan et al. J. Appl. Cryst. (2007). 40, 151-157. https://doi.org/10.1107/S0021889806047558

[50] J. Plomp et al. / Nuclear Instruments and Methods in Physics Research A 574 (2007) 324329. https://doi.org/10.1016/j.nima.2007.02.068

[51] M. Bech, O. Bunk, T. Donath, R. Feidenhans'l, C. David and F. Pfeiffer, Quantitative x-ray dark-field computed tomography, Phys. Med. \& Bio. 55, 18 (2010).

https://doi.org/10.1088/0031-9155/55/18/017

[52] 3. C. Grünzweig, J. Kopecek, B. Betz, A. Kaestner, K. Jefimovs, J. Kohlbrecher, U. Gasser, O. Bunk, C. David, E. Lehmann, T. Donath, and F. Pfeiffer, Quantification of the neutron darkfield imaging signal in grating interferometry, Phys. Rev. B 88, 125104, (2013).

https://doi.org/10.1103/PhysRevB.88.125104

[53] $\mathrm{PhD}$ thesis, M. Strobl, TU Wien 2003

[54] M. Strobl General solution for quantitative dark-field contrast imaging with grating interferometers. Scientific Reports 4 (2014) 7243. https://doi.org/10.1038/srep07243

[55] Andersson, R., van Heijkamp, L. F., de Schepper, I. M. \& Bouwman, W. G. Analysis of spin-echo small-angle neutron scattering. J. Appl. Cryst. 41, 868-885 (2008).

https://doi.org/10.1107/S0021889808026770

[56] J. Kohlbrecher and A. Studer, Transformation cycle between the spherically symmetric correlation function, projected correlation function and differential cross section as implemented in SASfit, J. Appl. Cryst. (2017). 50, 1395-1403. https://doi.org/10.1107/S1600576717011979

[57] M. Strobl, B. Betz, R. P. Harti, A. Hilger, N. Kardjilov, I. Manke, C. Gruenzweig Wavelength dispersive dark-field contrast: micrometer structure resolution in neutron imaging with gratings J. Appl. Cryst. 49 (2016). https://doi.org/10.1107/S1600576716002922

[58] K.M. Podurets, V.A. Somekov, R.R. Chistyakov and S.Sh.Shilstein, Visualization of internal domain structure of Silicon Iron crystals by using neutron radiography with refraction contrast, Physica B 156 \& 157 (1989) 694-697. https://doi.org/10.1016/0921-4526(89)90766-7

[59] I. Manke, N. Kardjilov, R. Schäfer, A. Hilger, M. Strobl, M. Dawson, C. Grünzweig, G. Behr, M. Hentschel, C. David, A. Kupsch, A. Lange, J. Banhart, Three-dimensionl imaging of magnetic domains, Nature Commun. 1, 125 (2010) . https://doi.org/10.1038/ncomms1125

[60] B. Betz, P. Rauscher, R. P. Harti, R. Schäfer, H. Van Swygenhoven, A. Kaestner, J. Hovind, E. Lehmann, and C. Grünzweig, Frequency-Induced Bulk Magnetic Domain-Wall Freezing Visualized by Neutron Dark-Field Imaging Phys. Rev. Applied 6, 024024 (2016). https://doi.org/10.1103/PhysRevApplied.6.024024

[61] M. Strobl et al. Appl. Phys. Lett. 91, 254104 (2007). https://doi.org/10.1063/1.2825276

[62] S. W. Lee, D. S. Hussey, D. L. Jacobson, C. M. Sim, M. Arif, Development of the grating phase neutron interferometer at a monochromatic beam line, Nuclear Instruments and Methods in Physics Research A 605 (2009) 16-20. https://doi.org/10.1016/j.nima.2009.01.225 